\section{Pore dynamics and asymmetric cargo loading in an encapsulin nanocompartment}

\author{
Jennifer Ross ${ }^{1,2}$, Zak Mclver ${ }^{3}$, Thomas Lambert ${ }^{1}$, Cecilia Piergentili ${ }^{3}$, Jasmine Emma Bird ${ }^{3}$, \\ Kelly J. Gallagher ${ }^{1}$, Faye L. Cruickshank ${ }^{1}$, Patrick James ${ }^{3}$, Efrain Zarazúa-Arvizu ${ }^{4}$, \\ Louise E. Horsfall ${ }^{4}$, Kevin J. Waldron ${ }^{2}$, Marcus D. Wilson ${ }^{5}$, C. Logan Mackay ${ }^{1}$, Arnaud Baslé ${ }^{2}$, \\ David J. Clarke ${ }^{1 *}$, Jon Marles-Wright ${ }^{2,3 *}$
}

\begin{abstract}
Encapsulins are protein nanocompartments that house various cargo enzymes, including a family of decameric ferritin-like proteins. Here, we study a recombinant Haliangium ochraceum encapsulin:encapsulated ferritin complex using cryo-electron microscopy and hydrogen/deuterium exchange mass spectrometry to gain insight into the structural relationship between the encapsulin shell and its protein cargo. An asymmetric single-particle reconstruction reveals four encapsulated ferritin decamers in a tetrahedral arrangement within the encapsulin nanocompartment. This leads to a symmetry mismatch between the protein cargo and the icosahedral encapsulin shell. The encapsulated ferritin decamers are offset from the interior face of the encapsulin shell. Using hydrogen/ deuterium exchange mass spectrometry, we observed the dynamic behavior of the major fivefold pore in the encapsulin shell and show the pore opening via the movement of the encapsulin A-domain. These data will accelerate efforts to engineer the encapsulation of heterologous cargo proteins and to alter the permeability of the encapsulin shell via pore modifications.
\end{abstract}

\section{INTRODUCTION}

Cellular metabolism and reaction pathways can produce toxic byproducts that damage proteins, DNA, and lipids or can become involved in potentially harmful side reactions. Eukaryotes use membranebound organelles, such as lysosomes, to prevent this damage by housing dangerous reactions in chemically privileged environments. In a similar manner, prokaryotes use large protein-based compartments to sequester such reactions and act as a barrier from the cytosol $(1,2)$. Prokaryotes have a variety of metabolic compartments including carboxysomes, which are used for carbon dioxide fixation in photosynthetic and some chemotrophic bacteria $(3,4)$; the related bacterial microcompartments, which allow the catabolism of carbon sources that produce aldehyde intermediates in their breakdown, such as ethanolamine and propanediol (5); and ferritins, used for iron oxidation and storage $(6,7)$.

One other key compartmentalization strategy used by prokaryotes and archaea is the encapsulin system (8-10). Encapsulin (Enc) nanocompartments are hollow icosahedral complexes that range in size from 20 to $42 \mathrm{~nm}(8,11,12)$. Encapsulin proteins are structurally related to the viral capsid protein (gp5) of the HK97 bacteriophage and self-assemble from a single monomer into one of three forms: 60 subunits ( $T=1$ capsid symmetry), 180 subunits $(T=3$ capsid symmetry), or 240 subunits ( $T=4$ symmetry) $(8,10,12,13)$. Encapsulins share a common feature of housing a cargo enzyme, such as ferritinlike proteins [encapsulated ferritins (EncFtn)], iron-mineralizing encapsulin-associated firmicute (IMEF), or dye-decolorizing

\footnotetext{
${ }^{1}$ EaStCHEM School of Chemistry, University of Edinburgh, Joseph Black Building, David Brewster Road, Edinburgh EH9 3FJ, UK. ${ }^{2}$ Newcastle University Biosciences Institute, Medical School, Newcastle University, Newcastle upon Tyne NE2 4HH, UK. ${ }^{3}$ School of Natural and Environmental Sciences, Newcastle University, Newcastle upon Tyne NE1 7RU, UK. ${ }^{4}$ School of Biological Sciences, University of Edinburgh, Edinburgh EH9 3BF, UK. ${ }^{5}$ Wellcome Centre for Cell Biology, University of Edinburgh, Michael Swann Building, Kings Buildings, Mayfield Road, Edinburgh EH9 3JR, UK. *Corresponding author. Email: jon.marles-wright1@ncl.ac.uk (J.M.-W.); dave.clarke@ ed.ac.uk (D.J.C.)
}

peroxidases $(9,12)$. Cargo enzymes are directed inside the encapsulin nanocompartment by a terminal localization sequence (LS) that binds to the interior face of the encapsulin $(8,14)$. Encapsulins and their cargo proteins are found throughout the bacterial and archaeal domains in species inhabiting a range of environmental niches; consequently, the proteins are stable in diverse physical conditions $(13,15-18)$. For these reasons, the encapsulins have attracted considerable interest for biotechnological applications through their ability to separate potentially hazardous heterologous reactions from the native cytosol $(19,20)$. The expression of functional engineered encapsulins has also been demonstrated in eukaryotes, where they have been developed as metabolic organelles in Saccharomyces cerevisiae (20) and in cellular imaging applications in human cells (21).

The EncFtn cargo proteins are of particular interest, as they differ from their classical ferritin relatives. Although both proteins oxidize iron using a conserved catalytically active ferroxidase center (FOC), they have remarkably different structural architectures. Classical ferritins oxidize ferrous iron, $\mathrm{Fe}(\mathrm{II})$, into a mineral ferric form, $\mathrm{Fe}(\mathrm{III})$, which is then stored within a 24 -meric 12 -nm nanocage $(6,22)$. In contrast to this, the EncFtn proteins have an annular structure formed from a pentamer of dimers with the FOC active sites located at a dimer interface $(11,15)$. EncFtn oxidizes iron in a similar manner to other ferritins, but due to its open structure, it must be associated with an encapsulin nanocage to act as an iron store (11). Together, the encapsulin EncFtn (Enc:EncFtn) complex can perform both the oxidation and storage functions of classical ferritins. However, due to its increased size when compared to classical ferritins, the Enc:EncFtn complex has the potential to house greater quantities of iron, and has been described as an iron megastore (13).

Although there have been several structural studies on encapsulins, several key questions remain unanswered. Most notably, for EncFtn containing encapsulin nanocompartments, the structural relationship between the encapsulin shell and the EncFtn cargo protein is unknown. Studies on Dyp-loaded encapsulin nanocompartments have 
shown loading with either one (14) or two hexameric complexes (23). Previous models for the loading of EncFtn in encapsulin nanocompartments have suggested a symmetric arrangement of the D5 decameric EncFtn at the fivefold icosahedral vertices of the encapsulin shell, giving a theoretical maximum of 12 EncFtn decamers per encapsulin nanocage $(11,24)$.

Here, we investigate the structure of the Enc:EncFtn nanocompartment from the halophilic bacterium Haliangium ochraceum to gain a better understanding of the arrangement and stoichiometry of the complex. Using the complementary structural biology techniques of cryo-electron microscopy (cryo-EM) and hydrogen/ deuterium exchange mass spectrometry (HDX-MS), we study the structural relationship between Enc and EncFtn in the encapsulin nanocage.

We present the first cryo-EM structure of a mesophilic Enc:EncFtn nanocompartment, which reveals a symmetry-breaking tetrahedral arrangement of the EncFtn decamers within the encapsulin shell. Analysis of the encapsulin shell by symmetry expansion of an icosahedral reconstructions and focused three-dimensional (3D) refinement on the pentameric vertex reveals a flexible pore in the shell. The dynamic nature of this pore region was investigated by HDXMS. We show that this region has a high rate of HDX, demonstrating its conformational flexibility. Our combination of HDX-MS and cryo-EM models affords insight into the loading capacity and dynamics of the Enc:EncFtn nanocompartment system.

\section{RESULTS}

\section{Recombinant $\boldsymbol{H}$. ochraceum encapsulin complexes form} regular nanocompartments that recruit active EncFtn cargoes To gain an understanding of the relationship between encapsulin nanocompartments and their EncFtn cargoes, the Enc:EncFtn nanocompartment from the halophilic mesophile $H$. ochraceum was chosen as our model system. This was primarily due to the high yields and ease of purification of the recombinant nanocompartment. Constructs to produce empty (Empty-Enc) and EncFtn-loaded (Loaded-Enc) encapsulin nanocompartments were assembled for recombinant protein expression in Escherichia coli. The protein complexes were purified by heat treatment, followed by anion exchange and size exclusion chromatography (SEC) (Fig. 1, A and B, and fig. S1, A to D).

SEC was used to separate correctly formed encapsulin nanocompartments from contaminating proteins and partially formed subcomplexes (fig. S1, A and B). Fractions corresponding to the major peaks were visualized by negative-stain transmission electron microscopy (TEM) (fig. S2), with the fraction corresponding to a retention volume of $63 \mathrm{ml}$ for both Empty-Enc and Loaded-Enc containing the homogeneous encapsulin nanocompartments (Fig. 1C). Both empty and EncFtn-loaded encapsulins assembled into regular nanocompartments, with an average diameter of approximately $21 \mathrm{~nm}$, consistent with other $T=1$ type encapsulins (fig. S1, E and F) $(8,23-25)$. The micrographs of the Loaded-Enc sample reveal a regular internal density visible within the nanocompartment, suggesting that the EncFtn cargo has been encapsulated in an organized manner.

The molecular masses of the protein constituents of the Empty-Enc and Loaded-Enc nanocompartments were determined by liquid chromatography-MS (LC-MS) (table S1). MS analysis of the EmptyEnc assembly revealed a single charge-state distribution corresponding
A

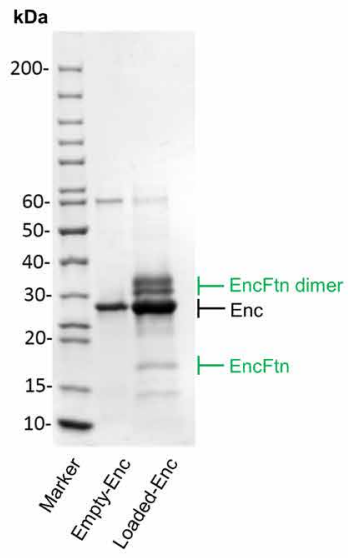

C
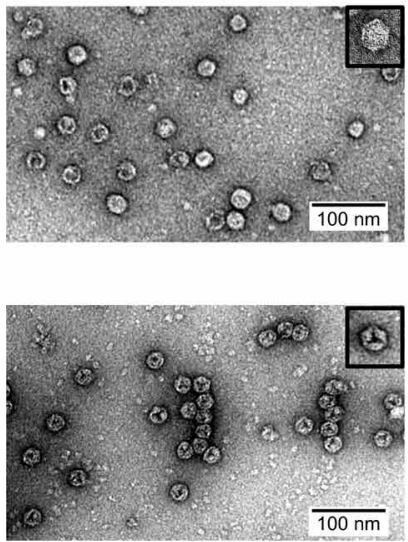

B

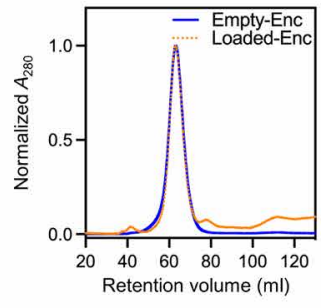

D
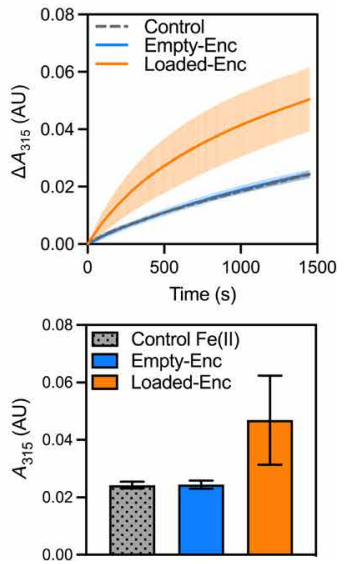

Fig. 1. Validation of the assembly and activity of Loaded-Enc and Empty-Enc nanocompartments. (A) SDS-polyacrylamide gel electrophoresis (PAGE) of purified Empty-Enc and Loaded-Enc. Proteins are resolved by 15\% acrylamide SDS-PAGE and stained with Coomassie blue stain. Encapsulin bands are near the 30-kDa marker and are highlighted by a black arrow. The EncFtn cargo of Loaded-Enc appears as both a monomer and a dimer. Bands corresponding to the monomer and dimer of EncFtn are highlighted with green arrows. (B) Recombinant, purified Empty-Enc (blue trace) and Loaded-Enc (orange, dotted trace) were analyzed by SEC using a Sephacryl 400 column (Cytiva). Both encapsulins elute at the same volume, suggesting that the encapsulin complexes are the same size regardless of cargo loading. (C) Negative-stain transmission micrographs of Empty-Enc (upper micrograph) and Loaded-Enc (lower micrograph) displaying individual particles for each complex. One nanocompartment of Empty-Enc and Loaded-Enc is shown in the upper right corner of each micrograph, with a hexagonal 2D geometry observed. (D) Top: Ferroxidase activity of Loaded-Enc compared to Empty-Enc. Protein samples were mixed with $100 \mu \mathrm{M} \mathrm{FeSO}_{4} .7 \mathrm{H}_{2} \mathrm{O}$. Following an incubation period at room temperature of $50 \mathrm{~s}$, absorbance at $315 \mathrm{~nm}$ was measured over a time course of $1450 \mathrm{~s}$. Control reference established using enzyme-free reaction as a measure of background iron oxidation. The lines represent the mean of all repeats; error bars represent the SD from the mean. Bottom: End point ferroxidase assay comparison. Ferroxidase activity shown by the total increase in $A_{315}$ (absorbance at $315 \mathrm{~nm}$ ) at the end point of the assay. Bars represent the mean of all repeats; error bars represent the SD from the mean. AU, Absorbance Units.

to a monomer of the encapsulin protein. MS analysis of the Loaded-Enc revealed three charge-state distributions present, with deconvoluted masses consistent with the encapsulin protein monomer, a monomer of EncFtn, and a dimer of EncFtn (fig. S1, G and H) (26). These 
results indicate that the Loaded-Enc sample contained both the encapsulin and EncFtn cargo proteins, while Empty-Enc has only the encapsulin protein.

Ferroxidase assays were performed on the samples to confirm the ability of the EncFtn-loaded encapsulin nanocompartment to convert $\mathrm{Fe}(\mathrm{II})$ to $\mathrm{Fe}$ (III) (Fig. 1D). This result is consistent with our previous observations for the Rhodospirillum rubrum Enc:EncFtn encapsulin complex (11). The empty encapsulin, which lacks the EncFtn cargo, is enzymatically inactive. Together, these data demonstrate that functionally active EncFtn has been successfully loaded into the encapsulin nanocompartment during expression in the heterologous E. coli host.

The cryo-EM structure of the Loaded-Enc nanocompartment Motivated by the apparent interior density in the Loaded-Enc sample, we performed single-particle cryo-EM on the Loaded-Enc nanocompartment complex (table S2). Consistent with previously published $\mathrm{x}$-ray crystallographic and cryo-EM-derived encapsulin models, an initial reconstruction was produced using Relion3.1 (27) with imposed I1 symmetry (Fig. 2, figs. S3 and S4, and table S3) $(8,11,13,25,28)$. This resulted in a reconstruction with a global resolution of $2.5 \AA$ as determined by the gold-standard Fourier shell correlation (FSC) at 0.143 (fig. S3D). The reconstruction displays a $T=1$ icosahedral arrangement of 60 encapsulin monomers, with clearly resolved secondary structure elements. Small pores are visible in the shell at, or close to, the two-, three-, and fivefold symmetry axes, where protein monomers interact with each other (Fig. 2A). Regions of the reconstruction around the icosahedral fivefold axes displayed a lower resolution than the other regions of the structure in a local resolution map (Fig. 2B). This is consistent with observations of local resolution maps for the Quasibacillus thermotolerans encapsulin reconstruction (12). The monomer of the encapsulin nanocompartment from the reconstruction displays a HK97-fold typical for encapsulins (Fig. 2C), with the position of the E-loop broadly determining the topology of the encapsulin nanocage. In the $T=1$ family I encapsulins, this loop is at $45^{\circ}$ to the boundary between the P- and A-domains (Fig. 2C), whereas, in the T $=7$ HK97 and $T=3$ and $T=4$ encapsulins, it is found in close apposition to the A-domain. The recently described family II encapsulin from Synechococcus elongatus (28) has an E-loop topology consistent with the $T=3$ and $T=4$ encapsulins but adopts a $T=1$ icosahedral nanocage. The shift in E-loop position relative to other $T=1$ encapsulins is a result of an extended N-terminal region, which extends beneath the loop; this forces the pentameric vertices into a raised morphology consistent with the larger encapsulin nanocages.

\section{The encapsulin nanocompartment recruits four EncFtn decamers to its lumen}

With the imposition of I1 symmetry on the reconstruction, the EncFtn cargo is not visible within the lumen. This suggests that the organization of the EncFtn protein within the encapsulin shell does not conform to icosahedral symmetry and is rotationally averaged through our symmetry-imposed processing. To gain insight into the structural relationship between the encapsulin shell and its EncFtn cargo protein, a reconstruction was produced with no imposed symmetry averaging (Fig. 3 and fig. S5). Separation of the dataset into five $3 \mathrm{D}$ classes revealed a highly populated class with amorphous density in the interior (fig. S4) and two other main classes, both containing four distinct densities, arranged in a similar tetrahedral
A

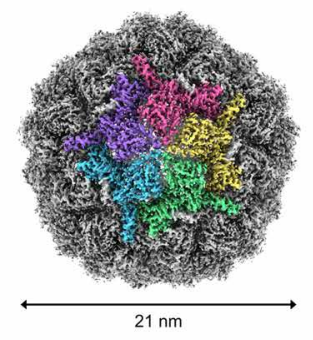

B



C

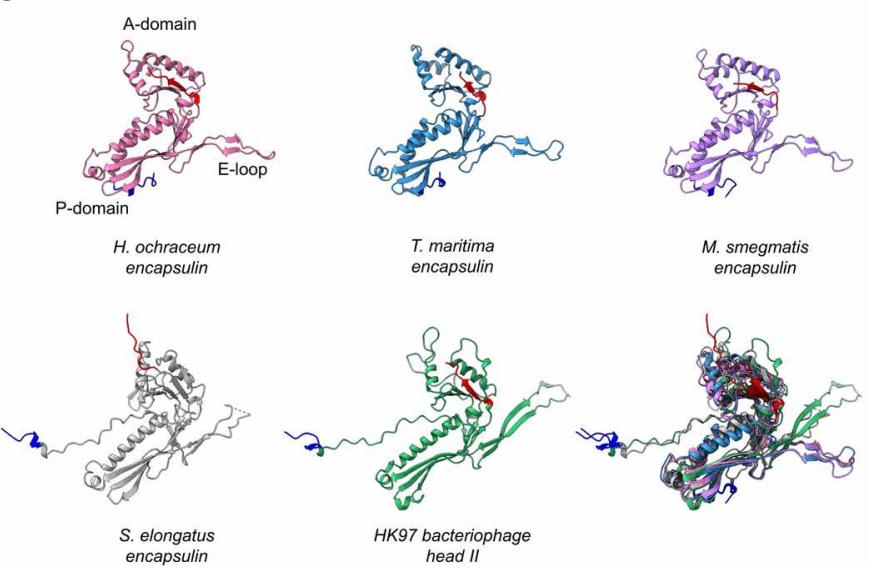

Fig. 2. Architecture of the $\boldsymbol{H}$. ochraceum encapsulin nanocompartment shell. Visualization of the electronic potential map of the $H$. ochraceum encapsulin from an icosahedrally averaged single-particle reconstruction. (A) Exterior of the encapsulin shell visualized at 2.4-Å resolution. Five subunits of the encapsulin nanocompartment shell have been colored to highlight the fivefold axis. (B) Icosahedral EM map of Loaded-Enc sharpened by local resolution estimate and colored by local resolution. The estimated resolution varies across the exterior of the encapsulin nanocompartment with the lowest resolution at the fivefold pores. Color key of resolution mapping is shown on the right-hand side of the figure. (C) Shared phage-like fold in the HK97 bacteriophage capsid and encapsulin proteins. Monomeric subunit of the $\mathrm{H}$. ochraceum encapsulin protein modeled from our reconstruction is shown (pink), with comparisons to other $T=1$ encapsulins from T. maritima [blue, Protein Data Bank (PDB) ID: 3DKT], Mycobacterium smegmatis (lilac, PDB ID: 7BOJ), and S. elongatus (gray, PDB ID: 6X8M). The HK97 bacteriophage head II $T=7$ monomer (green, PDB ID: 2FT1) is also shown. The $\mathrm{N}$ terminus of each monomer is highlighted in blue, and the $C$ terminus is highlighted in red. Bottom right: Overlay comparison of the encapsulin monomers showing similar A- and P-domain orientations.

fashion within the encapsulin shell. To obtain the clearest reconstruction of both the encapsulin nanocompartment and EncFtn cargoes, we took only one of these latter classes forward for full 3D refinement in Relion3.1 to produce a final $\mathrm{C} 1$ reconstruction with a resolution of $3.7 \AA$ at the 0.143 FSC threshold (Fig. 3A and fig. S5A). The EM map revealed four distinct densities within the encapsulin nanocompartment lumen, which are consistent in size and shape with four EncFtn decamers (Fig. 3B and fig. S5B).

A local resolution map calculated for the asymmetric reconstruction indicates a degree of flexibility at the pentameric pores compared to the trimeric pores of the encapsulin shell (Fig. 3A). The interior of the nanocompartment shows a significant falloff in resolution from the inner face of the encapsulin shell to the EncFtn 
A

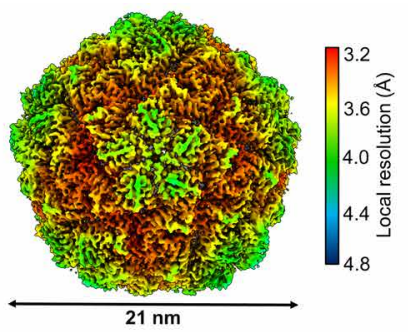

C

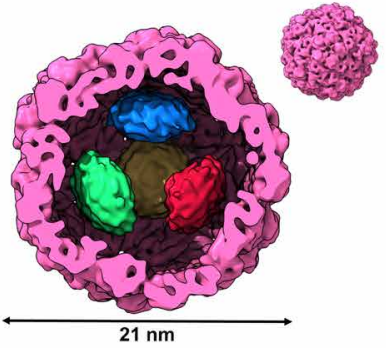

D

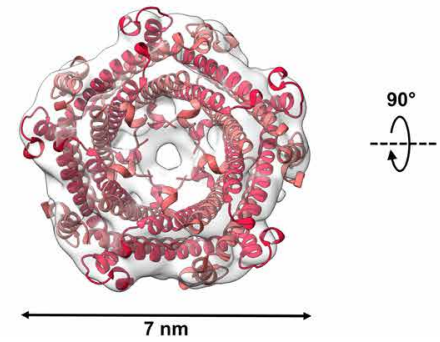

Fig. 3. Asymmetric reconstruction of the $\boldsymbol{H}$. ochraceum encapsulin complex reveals a tetrahedral arrangement of EncFtn within the encapsulin nanocompartment. (A) Electronic potential map of the asymmetric reconstruction of the H. ochraceum Enc:EncFtn complex. The map is colored by local resolution, with the color key shown on the right side of (A). (B) Radially colored cryo-EM-derived map of the Loaded-Enc nanocompartment displaying the interior EncFtn (cyan). (C) Gaussian smoothed C1 map showing the four discrete EncFtn densities (red, green, yellow, and blue), consistent with the size of decameric EncFtn complexes, within the encapsulin nanocompartment (pink). The four EncFtn are in a tetrahedral arrangement highlighted by gray lines connecting their centers of mass. The average distance between each EncFtn decamer is $57 \AA$. (D) Electronic potential map of a single EncFtn density following symmetry expansion and local refinement in CryoSPARC. The H. ochraceum EncFtn decamer crystal structure (PDB ID: 5N5F, red) is docked into the density.

densities. This is consistent with the tethering of EncFtn to the encapsulin nanocompartment via its LS, with some degree of conformational freedom of the EncFtn decamer with respect to the encapsulin shell.

The EncFtn decamers are located approximately $3 \mathrm{~nm}$ away from the encapsulin interior wall, which corresponds to the linker region between the main EncFtn domain and the LS on the EncFtn C terminus (fig. S5C). The extended LS of the EncFtn protein acts to offset it from the inner face of the encapsulin shell, an observation consistent with previous reports of the IMEF encapsulin complex from Q. thermotolerans (12). Because of the dynamic nature of EncFtn within the encapsulin, it was not possible to trace the path of the LS from the EncFtn to its binding site. The four EncFtn decamers are in a tetrahedral arrangement within the encapsulin nanocompartment, with the fivefold axes of the EncFtn decamers aligned to the threefold tetrahedral axes (Fig. 3C and fig. S5C). This results in a double symmetry mismatch between the icosahedral shell and the EncFtn decamers in the complex. A concurrent structural study of the Thermotoga maritima encapsulin complex (29) revealed five EncFtn decamers within the encapsulin shell, with each decamer found in approximation to a pentameric vertex. The overall arrangement of the five EncFtn complexes within the T. maritima nanocompartment is incompatible with the formation of a regular platonic solid and results in a symmetry-breaking arrangement of EncFtn within the encapsuling nanocompartment. To enhance the resolution of the individual EncFtn densities, symmetry expansion and local refinement focused on a single EncFtn density was performed in CryoSPARC. This resulted in an EM map into which the H. ochraceum EncFtn crystal structure [Protein Data Bank (PDB) ID: 5N5F] (15) could be docked (Fig. 3D).

Analysis of the relationship between the EncFtn decamers and the inner face of the H. ochraceum encapsulin shell reveals distinct EncFtn environments (fig. S6). The first EncFtn environment is shared by two EncFtn decamers and is in line with the fivefold pore of the encapsulin nanocompartment (EncFtn 1 and 2 in fig. S6). The shared symmetry of the Enc nanocompartment fivefold pores and of the EncFtn D5 annular structure in these positions is consistent with our previously proposed hypothesis for the Enc:EncFtn relationship (11) and is also found in the T. maritima encapsulin. However, the symmetry-breaking tetrahedral arrangement of the EncFtn decamers in the H. ochraceum encapsulin creates a second distinct environment shared by the remaining two EncFtn decamers, where they are offset between fivefold and threefold axes of the icosahedral encapsulin shell (EncFtn 3 and 4 in fig. S6). With the proposed route of iron entry through the fivefold pores of the encapsulin shell, the EncFtn decamers in proximity to the pores may have more favorable substrate access than those found in the alternative positions. Given the fast mass transport of substrates across the shell (30), this symmetry-breaking arrangement of the EncFtn decamers is unlikely to have significant functional consequences in terms of the speed of iron oxidation in the different EncFtn environments.

\section{Structural dynamics in the pentameric vertices of the encapsulin shell}

To further investigate the apparent conformational flexibility of the encapsulin shell at the pentameric vertices in the reconstructions, we analyzed the full cryo-EM dataset in CryoSPARC to take advantage of recent advances in $3 \mathrm{D}$ variability analysis (3DVA) of single-particle datasets (31). A C1 refinement calculated with 333,945 particles performed in CryoSPARC (table S4) was subjected to 3DVA with three variability components. The three components resolve to show the fivefold pores irising between "open" and "closed" states. The three components primarily differ in which pores are open/closed and show no other major differences. Adjacent fivefold pores show a mixture of correlated and anticorrelated opening (Fig. 4, fig. S7, and movies 1 to 3 ). This movement gives the impression of a pumping motion, which may enhance the transfer of substrates between the exterior and interior lumen of the nanocompartment.

Within the encapsulin nanocompartment, the EncFtn densities show some uncoordinated lateral motions, but their position relative to the inner face of the encapsulin does not change. This is because they are tethered by their LSs to the P-domain of the 
encapsulin, which does not show any significant displacement in any of the variability components.

To capture the extreme open and closed conformations of the fivefold pores, we performed symmetry expansion on the I1 particle set in Relion3.1, followed by masked 3D classification without alignment centered on the vertex. Of the five conformations observed, the most extreme of these were subjected to 3D refinement with local searches (Fig. 5 and figs. S8 and S9). This resulted in an open pentamer conformation of 2.4 - $\AA$ resolution and a closed conformation of $2.2 \AA$, allowing for fitting of residue side chains (fig. S7).

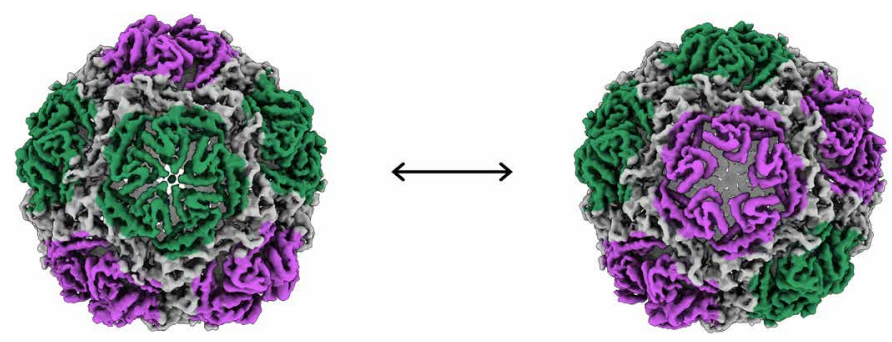

Fig. 4. 3D variability analysis of Loaded-Enc. The first and last frames from the trajectory of component 0 from the 3DVA are shown as density maps centered on a single fivefold pore. Pores across the encapsulin nanocompartment are seen to adopt conformations consistent with both closed (green) and open states (purple) and move between these along the trajectory. Different pores adopt this dynamic in the two other components calculated.
The open conformation has a fivefold pore with an aperture diameter of approximately $15 \AA$, while in the closed conformation the aperture is reduced to 5 - $\AA$ diameter. To understand the structural changes taking place in the transition between these conformations, an atomic model of the encapsulin protein was refined against both maps (table S5). The two models show a significant movement in the A-domain, with this region pivoting at the loops connecting it to the P-domain (residues 121 to 131 and 201 to 222) (fig. S10, A and B), opening the pore like an iris (Fig. 5). In the open conformation, the pore loop region (residues 182 to 189) is not well defined in the density; while it is tightly locked in the closed conformation, with $\mathrm{Asp}^{186}$ forming the outer boundary of the pore and $\mathrm{Tyr}^{188}$ and Lys $^{192}$ forming the inner bounds (fig. S10C). The tyrosine is well conserved among the family $1 T=1$ encapsulins, while the lysine is substituted for a glutamine in the R. rubrum encapsulin (fig. S11). The family $2 T=1$ encapsulin from $S$. elongatus has a five-residue sequence insertion in this region, which forms an extended linker between secondary structure elements, rather than a distinct loop within the pore.

In addition, our focused refinements of the pentameric subunits allowed us to build and sequence the ${ }_{117}$ GSLGIGSLR $_{125}$ peptide from the EncFtn protein (Fig. 6). This region of the LS forms a network of hydrophobic interactions with the inner face of the P-domain of a single encapsulin monomer, with further stabilization by several water-mediated backbone contacts. The core GxLGIxxL motif found in this region of the LS is conserved between the H. ochraceum

A

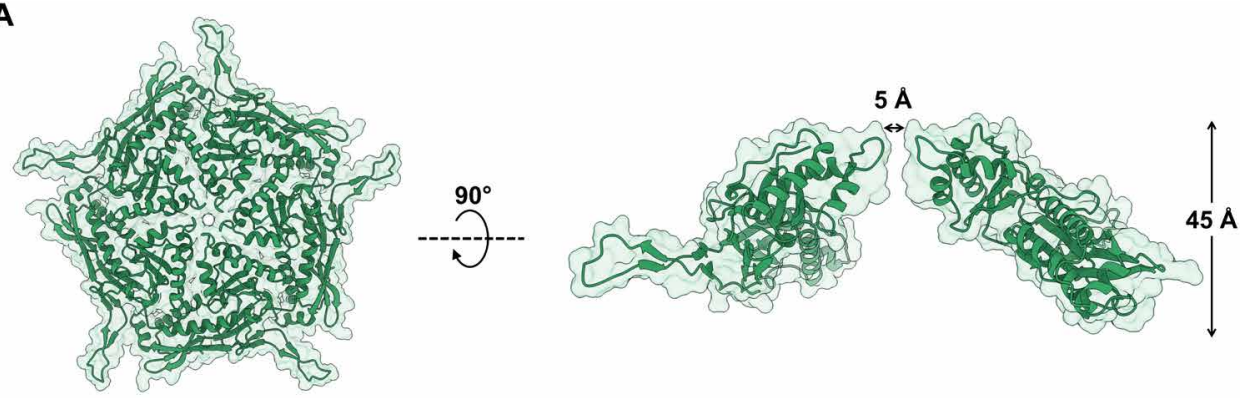

B

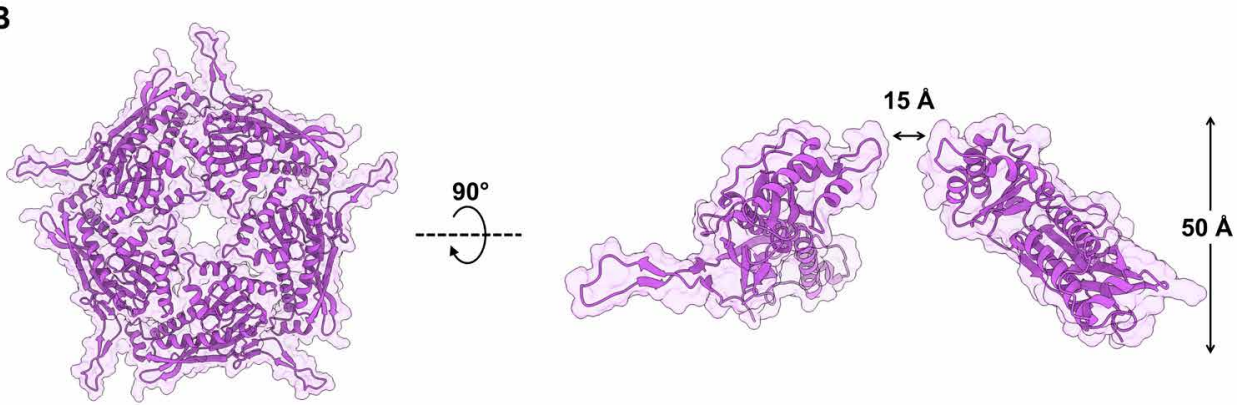

Fig. 5. Open and closed conformations of the dynamic fivefold pore of the $\boldsymbol{H}$. ochraceum encapsulin shell. Masked 3D refinements centered on the fivefold pore of the icosahedral reconstruction were performed after symmetry expansion of the asymmetric units. The closed [(A), green] and open [(B), purple] conformations of the Loaded-Enc shell pentamer are represented with transparent solvent-accessible surface rendered over cartoon secondary structure. Both closed and open pentamers are shown in top-down (left-hand side) and side-on views (right-hand side). The closed conformation has a fivefold pore diameter of $5 \AA$, while the equivalent diameter of the open conformation is $15 \AA$. To allow for the widening of the pore, the open pentamer protrudes from the nanocompartment by $5 \AA$. 
A

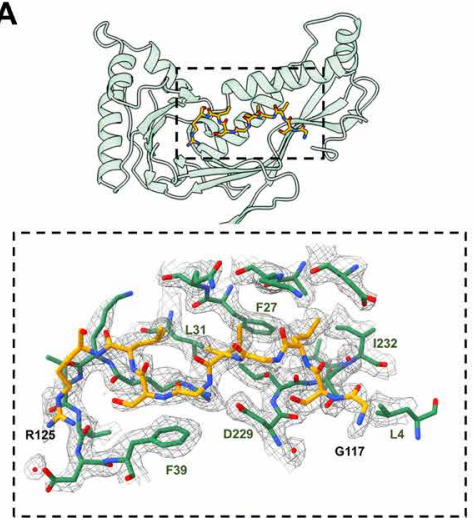

B

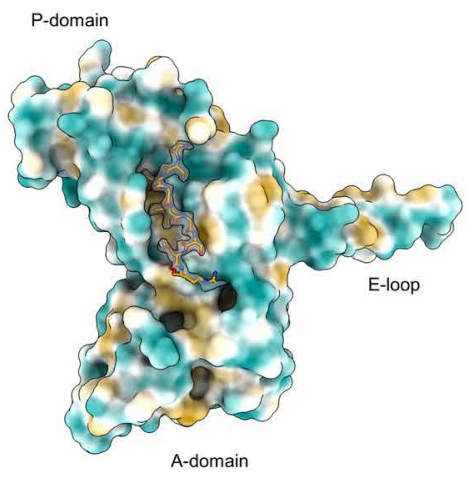

C

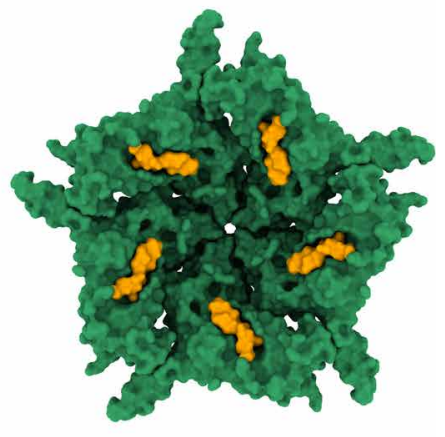

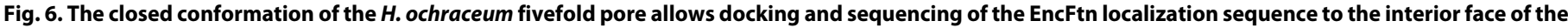

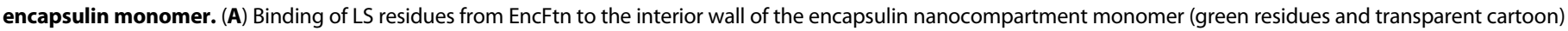

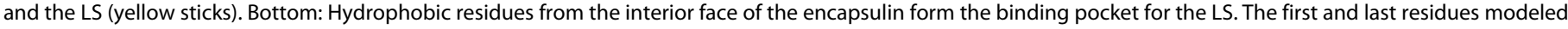

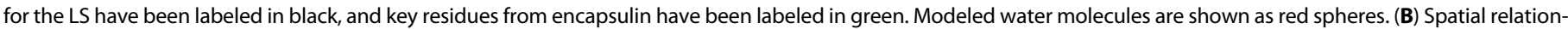

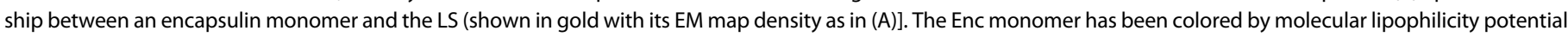

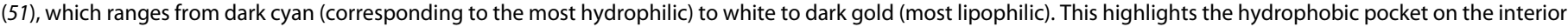
of the encapsulin nanocompartment where the LS binds. (C) Closed conformation encapsulin pentamer (green), with an LS (yellow) shown on each monomer.

EncFtn and other proteins in the family and is observed in the crystal structure of the T. maritima encapsulin (8).

In the H. ochraceum encapsulin, the fivefold pore has a negative charge on the exterior of the encapsulin shell and positive charge on the interior in both the open and closed conformations (fig. S12). No changes are observed in the structure, or charge of the three- and twofold pores (fig. S12). The closed conformation of the fivefold pore is consistent with observations from the crystal structure of the T. maritima encapsulin (8) and high-resolution cryo-EM structures of other encapsulins $(12,30,31)$. However, this is the first time that an open pore conformation has been observed in an encapsulin protein.

This observation has important implications for efforts to engineer the pores of encapsulin nanocages. Efforts to widen the fivefold pores have demonstrated an increase in mass transport of model substrates and ions across the encapsulin shell $(30,32)$; however, the observed changes in pore diameters are limited to a maximum of around $10 \AA$ in the T. maritima encapsulin (32). The encapsulin shell appears to interact strongly with $\mathrm{Tb}^{3+}$ ions in molecular dynamics simulations; this is consistent with our previous observations of iron binding by the R. rubrum encapsulin (11) and highlights a possible cooperative role for substrate channeling via the encapsulin shell to the EncFtn cargoes.

Our results suggest that the flexibility of the A-domain should also be considered when engineering the pore structure for enhanced transport across the encapsulin shell. The loops connecting the A-domain to the P-domain present ideal sites for targeted mutagenesis to stiffen, or lock, the position of the A-domain and thus constrain the fivefold pore loop to engineer enhanced substrate selectivity into these systems.

\section{Dynamics of the fivefold encapsulin pore through hydrogen/ deuterium exchange mass spectrometry}

To further investigate the dynamic nature of the fivefold pore of the encapsulin shell and the docking of the EncFtn LS to the interior of the encapsulin nanocompartment, we performed HDX-MS on both Empty-Enc and Loaded-Enc nanocompartments. The extent of backbone-amide hydrogen exchange was determined at seven time points ( $0 \mathrm{~s}, 10 \mathrm{~s}, 30 \mathrm{~s}, 5 \mathrm{~min}, 30 \mathrm{~min}, 4$ hours, and 24 hours).
By calculating the rate of hydrogen exchange throughout the protein, regions that differ in solvent exposure and/or dynamics can be detected.

HDX-MS analysis of the encapsulin nanocompartment resulted in 40 pepsin peptides, which constituted a protein sequence coverage of $85 \%$, with peptide redundancy of 2.28 (fig. S13 and tables S6 and S7). The encapsulin nanocompartment displayed variable exchange rates throughout the protein sequence, and regions of the protein displaying elevated HDX rates were evident. Overlaying these local HDX rates onto the cryo-EM reconstruction revealed that the regions of highest exchange were located around pentameric vertices (Fig. 7). This was most notable with the peptide spanning the region between amino acids 180 to 196, which includes the fivefold pore loop (fig. S14). In contrast, lower rates of HDX are observed at the twofold interface and the potential threefold pore (Fig. 7). These findings agree with our cryo-EM structural analyses and support the proposed conformational flexibility at the fivefold pore.

Comparison of the HDX rates of Empty-Enc and Loaded-Enc revealed similar exchange profiles throughout the encapsulin protein sequence, suggesting that cargo loading has little effect on the overall architecture and dynamics of the assembled nanocompartment shell (fig. S15 and tables S6 and S7). However, after prolonged exchange times (4 hours), the Loaded-Enc exhibited areas with a modest reduction in exchange when compared to Empty-Enc. Notably, several peptides in the N-terminal region displayed reduced exchange rates in Loaded-Enc; for example, the peptide covering amino acids 21 to 37 displayed almost $12 \%$ reduction. Mapping the position of this region onto our encapsulin reconstruction highlights that this peptide is located on the interior face of the nanocompartment and included the proposed binding site for the LS of EncFtn (Fig. 7). A reduction in exchange across in this region is likely a consequence of shielding by the engaged EncFtn LSs.

\section{DISCUSSION}

Our cryo-EM reconstruction of an Enc:EncFtn nanocompartment complex reveals key areas of divergence from a true icosahedral 

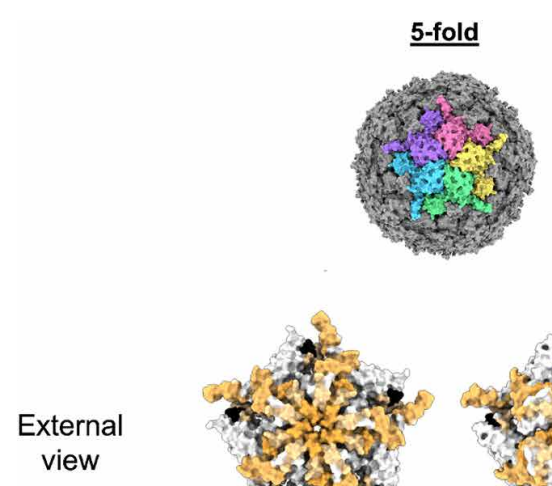

Internal

view
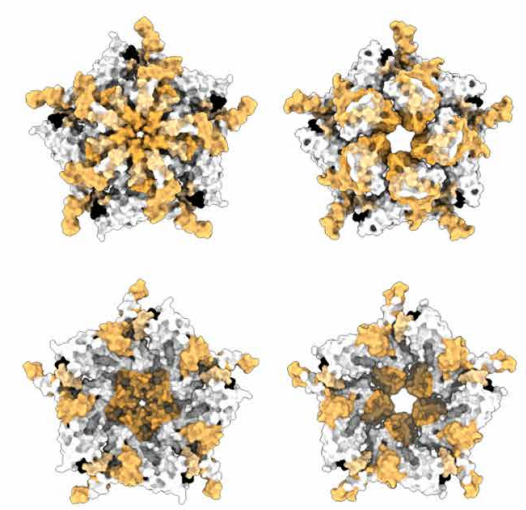
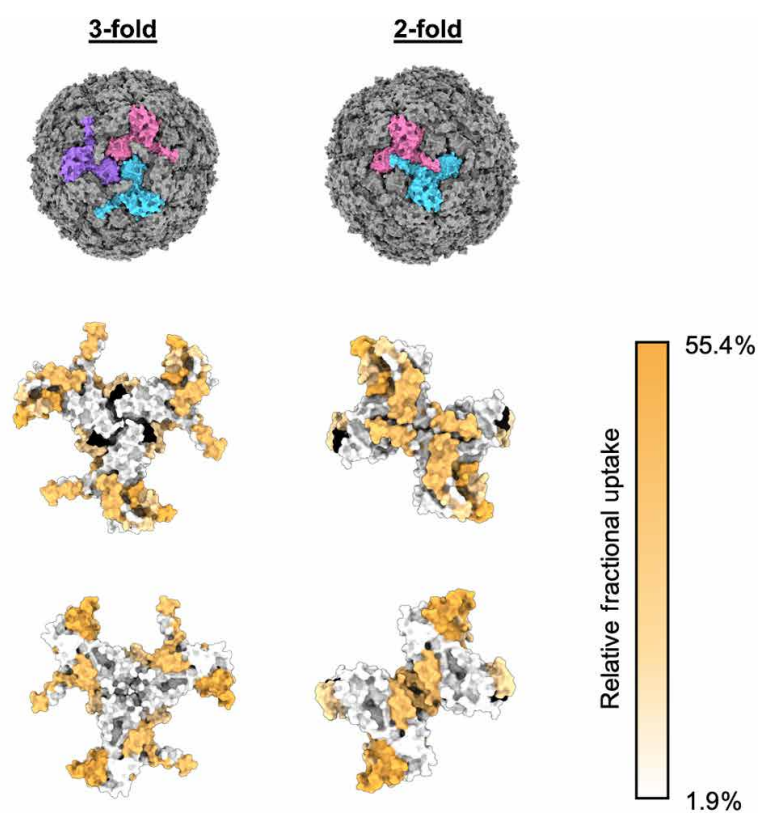

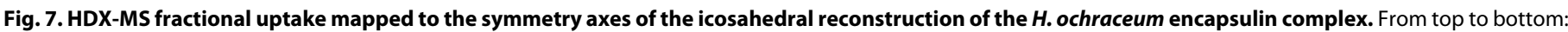

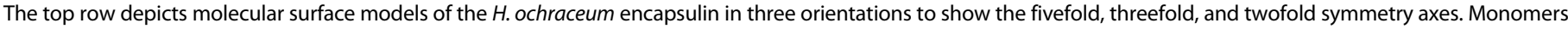

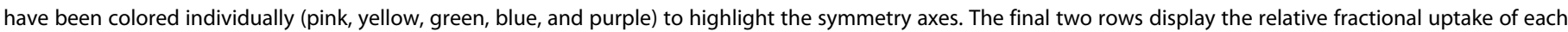

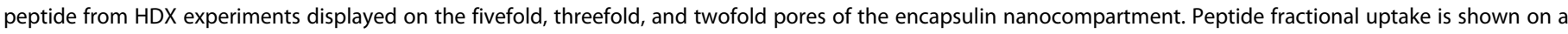
white-to-orange color scale, with a color key shown on the right-hand side of the figure. Areas colored black correspond to no peptide coverage.

complex with important functional consequences. The asymmetric reconstruction showed that the encapsulin nanocompartment sequesters four decamers of EncFtn within its lumen. In our recombinant system, with EncFtn produced in excess, this likely represents a maximum loading capacity for these Enc:EncFtn nanocompartments. The symmetry-breaking tetrameric arrangement of the EncFtn decamers within the encapsulin shell leads to two distinct environments for EncFtn, with two decamers aligned at the fivefold symmetry axes, and the remaining two residing between three- and fivefold axes (fig. S6). The concurrent observation of five EncFtn decamers within the T. maritima encapsulin nanocompartment, aligned close to the icosahedral fivefold symmetry axes of the encapsulin shell, highlights differences in cargo loading between encapsulins from mesophilic and thermophilic bacteria (29). The enhanced cargo loading seen in the T. maritima encapsulin may be a consequence of a more compact and rigid structure adopted by thermophilic proteins, as seen in comparisons of the crystal structures of the H. ochraceum and Pyrococcus furiosus EncFtn proteins (15).

The interior volume of the $T=1$ encapsulin lumen is around $4000 \mathrm{~nm}^{3}$, while an EncFtn decamer is only $120 \mathrm{~nm}^{2}$; therefore, the additional cargo seen in the T. maritima encapsulin should not significantly affect the iron-storage potential. The order of magnitude discrepancy in the iron-loading capacity measured for the $R$. rubrum (11) and T. maritima (29) encapsulins is likely a result of differences in experimental conditions in different laboratories.

While the volume occupied by the EncFtn decamers represents less than $15 \%$ of the total lumen of the encapsulin nanocompartments, the consistent observation of a gap between the encapsulin shell and encapsulin cargo proteins implies that the loading and capacity of encapsulins is limited by broader steric effects. Further steric effects would also be found from the unengaged LSs at the core of the nanocompartment. These steric effects have implications for efforts to target heterologous proteins to the encapsulin nanocage, effectively setting a limit on the volume of protein that can be accommodated within, which is much lower than the total volume of the lumen of the nanocage.

Our observations have functional implications for the oxidation and storage of iron within the Enc:EncFtn nanocompartment. The EncFtn decamers are in nonequivalent positions and thus have different relationships to the pores of the encapsulin shell. Therefore, if the pores limit the diffusion of substrates, the EncFtn decamers would be subjected to different chemical environments. Furthermore, both the engaged and unengaged LSs present a "soft" steric barrier to the diffusion of substrates. It is notable that the ferroxidase activity of the Enc:EncFtn complex is significantly higher than the isolated EncFtn protein (33). The dynamic behavior of the encapsulin fivefold pores, which is apparent in our 3DVA movies, may increase EncFtn activity by enhancing diffusion of substrates into the lumen of the nanocompartment. While it is not possible to make mechanistic conclusions from our model, the complex interactions with the components of the encapsulin nanocompartment clearly enhance the iron oxidation activity of the EncFtn protein.

Our data were collected on iron-free apo-Enc:EncFtn complexes, and thus, it is not possible to infer the nature of the iron mineralization pathway within the encapsulin nanocage. Further careful work must be performed to titrate iron into the complex before structural analysis. This will allow us to gain insight into the flow of metal ions from the exterior to the interior of the encapsulin and to determine whether metalation influences the conformational flexibility of EncFtn within the encapsulin nanocage, as we have demonstrated for isolated EncFtn proteins $(11,15,34)$. Last, the nature of the iron mineral and its localization within the encapsulin nanocage is still to be determined. 
These cryo-EM and HDX-MS data, illustrating a highly dynamic fivefold pore in the encapsulin shell, have major implications for efforts to engineer recombinant encapsulins for improved access for both native and nonnative substrates. The limitations of previously published studies, where the fivefold pore is modified for altered substrate access, can be explained by a highly dynamic pore structure. Our work suggests testable hypotheses for engineering pore selectivity, through modifications to the hinge regions between the $\mathrm{P}$ - and A-domains, which are responsible for the opening of the pore.

\section{MATERIALS AND METHODS Experimental design}

The objective of this study was to understand the structural relationship between encapsulins and their EncFtn cargo using cryo-EM to determine the complex structure and ferroxidase assays for validation of the complex activity. HDX-MS was used to establish differences between solvent accessibility of empty and loaded encapsulins and garner insight into the impact of cargo on the interior of the encapsulin nanocompartment.

\section{Cloning of encapsulin expression constructs}

The H. ochraceum encapsulin and EncFtn protein expression constructs were based on the Hoch_3836 and Hoch_3837 genes downloaded from www.kegg.jp and were codon-optimized for expression in E. coli and synthesized as CIDAR MoClo compatible gBlocks by Integrated DNA Technologies (IDT) (table S8). The gBlocks were assembled into a Level 0 CIDAR MoClo storage vector (35), DVA_CD, for subsequent use. The coding sequences for the encapsulin and EncFtn were assembled into expression cassettes in the level 1 backbones DVK_AE and DVK_EF, respectively, each with T7 promoter and transcription terminator parts. The resulting expression cassettes were then combined into the DVA_AF backbone to produce a coexpression plasmid. All assembled plasmids were sequence-verified by Sanger sequencing by Eurofins Genomics. The protein sequences for each construct are listed in table S9.

\section{Protein expression}

The Empty-Enc and Loaded-Enc expression plasmids were transformed into E. coli BL21(DE3) cells and grown overnight at $37^{\circ} \mathrm{C}$ on $\mathrm{LB}$ agar plates containing appropriate selection antibiotics (kanamycin for Empty-Enc and ampicillin for Loaded-Enc). A single colony of cells was added to 1 liter of autoinduction medium (36) supplemented (table S10) with appropriate antibiotic and grown for 38 hours at $37^{\circ} \mathrm{C}$ with shaking at $200 \mathrm{rpm}$. Cells were harvested by centrifugation at $12,000 g$.

\section{Encapsulin nanocompartment purification}

E. coli cell pellets expressing the Empty-Enc and Loaded-Enc constructs were resuspended in $10 \times \mathrm{v} / \mathrm{w}$ of lysis buffer $[20 \mathrm{mM}$ Hepes ( $\mathrm{pH} 8), 2 \mathrm{mM} \mathrm{MgCl} 2$, lysozyme $(1 \mathrm{mg} / \mathrm{ml})$, and benzonase (12.5 to $25 \mathrm{U} / \mathrm{ml})$ ]. Cells were lysed by sonication while on ice; sonication was carried out in six 1-min cycles (30-s sonication and 30-s rest). The lysate was clarified by centrifugation at $20,000 \mathrm{~g}$ for 1 hour $4^{\circ} \mathrm{C}$.

The supernatant from cell lysis was heated to $85^{\circ} \mathrm{C}$ for $10 \mathrm{~min}$ in a water bath and transferred to a $4^{\circ} \mathrm{C}$ ice bath for $10 \mathrm{~min}$. The supernatant was then collected after centrifugation at $10,000 \mathrm{~g}$ for 1 hour.

Anion exchange chromatography of the clarified supernatant was performed using a 1-ml HiTrap Q Sepharose FF column from
Cytiva on an ÄKTA start. The column and ÄKTA start system were equilibrated with QA buffer (20 mM Hepes, $\mathrm{pH} 8.0$ ), and the protein sample was loaded. Unbound proteins were removed by washing with QA buffer. Bound proteins were eluted by QB buffer [20 mM Hepes $(\mathrm{pH} 8.0)$ and $1 \mathrm{M} \mathrm{NaCl}$ ] over a linear gradient of 0 to $100 \% \mathrm{QB}$ over 15 column volumes. Flow-through fractions containing the sample were subjected to $15 \%$ SDS-polyacrylamide gel electrophoresis (PAGE) to identify those containing the protein of interest. These fractions were pooled and concentrated using centrifugal concentrators with a $30-\mathrm{kDa}$ nominal molecular weight cutoff (Vivaspin).

Pooled and concentrated samples from the anion exchange step were loaded on a SEC column (Sephacryl 400, Cytiva) equilibrated with SEC buffer [20 mM Hepes (pH 8.0) and $150 \mathrm{mM} \mathrm{NaCl}$ ]. Fractions eluting from the column containing the desired protein, as identified by SDS-PAGE, were pooled and concentrated as above. Protein aliquots were flash-cooled in liquid nitrogen and stored at $-80^{\circ} \mathrm{C}$ (fig. S1, A, i and ii, and $\mathrm{B}, \mathrm{i}$ and ii) $(11,15)$.

Following purification, aliquots of Empty-Enc and Loaded-Enc were subjected to a second round of polishing SEC using a Sephacryl 400 column (Cytiva) equilibrated with SEC buffer [20 mM Hepes (pH 8.0) and $150 \mathrm{mM} \mathrm{NaCl}$ (Fig. 1B) (experimental data for the SEC runs are available at doi:10.6084/m9.figshare.16698106).

\section{Negative-stain TEM}

Purified encapsulin nanocompartments were initially imaged by negative-stain TEM. Continuous carbon/formvar-coated copper grids (200 mesh) were glow-discharged for 30 s using a Pelco glow discharge system. Five microliters of Enc was pipetted onto the glow-discharged grids, and excess liquid was removed after $30 \mathrm{~s}$ with Whatman filter paper (grade 1; diameter, $24.0 \mathrm{~cm}$ ). The grids were washed with distilled water three times, followed by staining with $2 \%$ uranyl acetate for $5 \mathrm{~s}$. Grids were left to air dry and then imaged with a JEOL JEM-1400 transmission electron microscope. Images were collected with a Gatan CCD OneView camera and analyzed using FIJI (37) (raw negative-stain images are available at doi:10.6084/m9.figshare.16698088).

\section{Ferroxidase activity assay}

The enzymatic activity of Empty-Enc and Loaded-Enc was assessed by ferroxidase assay, as previously described (Piergentili, 2020). Fe(II) samples were prepared by dissolving $\mathrm{FeSO}_{4} .7 \mathrm{H}_{2} \mathrm{O}$ in $\mathrm{HCl} 0.1 \%(\mathrm{v} / \mathrm{v})$ under anaerobic conditions. Protein samples were diluted anaerobically in buffer GF [20 mM Hepes ( $\mathrm{pH} 8.0$ ) and $150 \mathrm{mM} \mathrm{NaCl}$ ] to a final encapsulin monomer concentration of $9 \mu \mathrm{M}$ to allow comparison between experiments.

Iron and protein aliquots were added aerobically to a quartz cuvette (Hellma), resulting in a final concentration of $100 \mu \mathrm{M}$ iron and $15 \mu \mathrm{M}$ Loaded-Enc or $9 \mu \mathrm{M}$ Empty-Enc. The cuvette was placed in a ultraviolet-visible spectrophotometer (PerkinElmer Lambda 35), and the reaction sample was incubated at $21^{\circ} \mathrm{C}$ for $50 \mathrm{~s}$ to stabilize. Absorbance at $315 \mathrm{~nm}$ was then recorded every second for $1450 \mathrm{~s}$ using the Time-Drive software. A control experiment was conducted by monitoring the background oxidation by atmospheric oxygen of $100 \mu \mathrm{M} \mathrm{FeSO}_{4}{ }^{*} 7 \mathrm{H}_{2} \mathrm{O}$ in the absence of the enzyme. Loaded-Enc experiments were carried out with three biological replicates. There were six technical replicates for batch one, two replicates using batch two, and one replicate from batch three. Means and SDs were calculated on the time zero-subtracted progress curves (experimental 
data for the ferroxidase assays are available at doi:10.6084/m9. figshare.16698127).

\section{Liquid chromatography-mass spectrometry}

LC-MS experiments were performed on a Synapt G2 Q-ToF instrument (Waters Corp., Manchester, UK) and an ACQUITY UPLC equipped with a reverse-phase C4 Aeris Widepore $50 \times 2.1 \mathrm{~mm}$ HPLC column (Phenomenex, CA, USA). Mobile phases of $\mathrm{A}=$ water + $0.1 \%$ formic acid and $\mathrm{B}=$ acetonitrile $+0.1 \%$ formic acid were used on a 10 -min gradient from $5 \%$ B to $95 \%$ B. Samples were analyzed at $\sim 2 \mu \mathrm{M}$, and data analysis was performed using MassLynx v4.1 and MaxEnt deconvolution.

\section{Cryo-EM data collection and analysis Sample vitrification}

Holey grids (gold, 200 mesh, r 2/2 by Quantifoil) were glow-discharged for $30 \mathrm{~s}$ using a Pelco glow discharge system. The grids were then mounted into a FEI vitrobot, and $4 \mu \mathrm{l}$ of encapsulin sample $(3 \mathrm{mg} / \mathrm{ml})$ was applied. Grids were then blotted $\left(100 \%\right.$ humidity, $8^{\circ} \mathrm{C}$, blot force -5 , wait time $10 \mathrm{~s}$, and blot time $3 \mathrm{~s}$ ) with Whatman filter paper (grade 1), flash-cooled in liquid ethane, and cooled with liquid nitrogen.

\section{Cryo-EM data collection}

Cryo-EM grid screening was performed on a FEI F20 microscope equipped with a FEG electron source $(200 \mathrm{kV})$ and a TVIPS F816 CMOS detector at the University of Edinburgh. The dataset used for single-particle reconstruction was obtained at the Electron Bioimaging Centre (eBIC) on a FEI Titan Krios microscope equipped with a Gatan K3 camera (data collection settings are shown in table S2). Alignments, grid transfer, and imaging setup were performed by the eBIC local contact Y. Song (cryo-EM movies are available in the EMPIAR database: EMPIAR-1218).

\section{Single-particle reconstruction}

Processing steps for the main reconstruction were performed with the Relion3.1 software package (28). Super-resolution movies were binned at $2 \times 2$ pixels for both $\mathrm{I} 1$ and $\mathrm{C} 1$ and motion-corrected using MotionCor2 (37). Defocus values of images (summed movies) were determined by CTFFIND4 (38), and those with poor CTF (contrast transfer function) fits, or bad ice, were manually discarded. A template for autopicking was created using $2 \mathrm{D}$ classes from manually picked particles. Autopicked particles were extracted (using a box size of 512 pixels) and subjected to three rounds of $2 \mathrm{D}$ classification to remove bad particles. An initial 3D model was created from particles selected from 2D classes. 3D classes were generated both with and without icosahedral symmetry imposed ("I1" and "C1" symmetry). The best class from each was taken forward for 3D refinement and then CTF refinement followed by further rounds of 3D refinement and Bayesian polishing. After a final round of 3D refinement, postprocessing was performed using a soft spherical mask. Local resolution estimation was performed in Relion3.1. The data processing and refinement pipeline is shown in fig. S3, with data processing and refinement statistics in table S3.

3DVA was performed using CryoSPARC $(31,39)$. Super-resolution movies were patch motion-corrected and patch CTF-estimated. Initially, particles were picked using blob picker (250 to $300 \AA$ ), and these particles were subjected to 2D classification followed by a round of ab initio model generation to create an autopick template. Successive rounds of 2D classification were performed on the autopicked particles until less than $5 \%$ of the particles were rejected. Five volumes were created by the ab initio model generation algorithm, and these were heterogeneously refined. The volume with the most particles (99.4\%) was homogeneously refined and subsequently subjected to 3DVA (31). The homogenously refined particles were also used to enhance the resolution of the EncFtn cargo. Particles were subjected to symmetry expansion, and then an individual EncFtn was masked and subjected to local refinement. The data processing and refinement performed in CryoSPARC pipeline is shown in fig. S4, with data processing and refinement statistics in table S4. Movies were created with ChimeraX (40).

Motivated by the dynamic behavior of the fivefold pores of the encapsulin shell, the fivefold pore pentamer was subjected to symmetry expansion and focused classification. The I1 particle set was expanded using the sym_expand job in Relion3.1, and masked 3D classification without alignment was performed focusing on the fivefold symmetry axis. Of the five classes produced, the two most highly populated and distinct were taken forward for masked 3D refinement with local searches only; these two classes represent the open and closed conformation of the fivefold pore. The mask used in these steps was produced in Chimera using the molmap command on a docked model of a pentamer of the encapsulin protein. To ensure that the box size and pixel size of the mask were correct, they were resampled onto the icosahedral map using the vop resample command.

\section{Model building and refinement}

An initial homology model of the encapsulin nanocompartment monomer was generated using Phyre 2.0 (41) based on the T. maritima structure. This was docked into the open and closed maps using ChimeraX (42) and expanded to a full pentamer model. The model was then fit to the map through an iterative process of automated model refinement with phenix real-space refinement (43) and manual model building in Coot (44), and waters were added using phenix. douse and validated in Coot. The resulting models and maps were validated using MolProbity (45), phenix.mtriage (46), and EM ringer (table S2) (47). Models and maps were visualized using ChimeraX.

\section{Encapsulin sequence analysis}

Encapsulin sequences were obtained from the Kyoto Encyclopedia of Genes and Genomes (www.kegg.jp). Sequence alignments were performed using Clustal Omega (48) and visualized using ESPript (49).

\section{Hydrogen/deuterium exchange mass spectrometry}

HDX-MS was performed on a Synapt G2 MS system coupled to an ACQUITY UPLC M-Class UPLC with the HDX manager module (Waters Corporation, Manchester, UK) (50). For improved reliability and precision, a custom-built Leap automated platform was used in all sample preparation and injections. Before HDX-MS analysis, three buffer solutions were prepared: (i) equilibration buffer $(4.7 \mathrm{mM}$ $\mathrm{K}_{2} \mathrm{HPO}_{4}$ and $0.3 \mathrm{mM} \mathrm{KH}_{2} \mathrm{PO}_{4}$ in $\mathrm{H}_{2} \mathrm{O}$ ), adjusted to $\mathrm{pH} 8.0$ with formic acid; (ii) labeling buffer $\left(4.7 \mathrm{mM} \mathrm{K}_{2} \mathrm{HPO}_{4}\right.$ and $0.3 \mathrm{mM}$ $\mathrm{KH}_{2} \mathrm{PO}_{4}$ in $\mathrm{D}_{2} \mathrm{O}$ ), adjusted to $\mathrm{pH} 8.0$ with $\mathrm{DCl}$; and (iii) quench buffer $\left(50 \mathrm{mM} \mathrm{K}_{2} \mathrm{HPO}_{4}\right.$ and $50 \mathrm{mM} \mathrm{KH}_{2} \mathrm{PO}_{4}$ in $\left.\mathrm{H}_{2} \mathrm{O}\right)$, adjusted to $\mathrm{pH} 2.3$ with formic acid. Protein samples were diluted in equilibration buffer to a final stock concentration of $42 \mathrm{mM}$. The time course experiments consisted of seven time points: T0 (0 min; undeuterated control), T1 (20 s), T2 (30 s), T3 (2 min), T4 (5 min), T5 (30 min), T6 (4 hours), and T7 (24 hours), with each time point being performed in triplicate. Sample preparation consisted of $5 \mu$ l of protein solution and $57 \mu \mathrm{l}$ of equilibration buffer (T0) or labeling buffer (T1 to T7). The final concentration of deuterium during the labeling 
step was $91.2 \%$. Exchange was allowed to proceed at $4^{\circ} \mathrm{C}$. To arrest the exchange reaction, $50 \mu$ of quench buffer was added to this initial solution just before sample injection.

After injection, samples underwent proteolytic digestion on a $2.1 \times 30 \mathrm{~mm}$ Waters Enzymate $\mathrm{BEH}$ pepsin column for $3 \mathrm{~min}$ at $200 \mu \mathrm{l} / \mathrm{min}$. After digestion, the peptide digest was loaded onto a $2.1 \times 5.0 \mathrm{~mm}$ Acquity BEH C18 VanGuard 1.7- $\mu \mathrm{m}$ C18 Trapping column to preconcentrate the sample for $3 \mathrm{~min}$ at $200 \mu \mathrm{l} / \mathrm{min}$. Following trapping, the digests were separated through a $2.1 \times 5.0 \mathrm{~mm}$ Acquity BEH 1.7- $\mu \mathrm{m}$ analytical column before MS/MS $\left(\mathrm{MS}^{\mathrm{e}}\right)$ analysis via the Waters Synapt G2 MS system running MassLynx v4.1 software (Waters Corporation, Manchester, UK). The separation gradient was 5 to $95 \%$ acetonitrile with $0.1 \%$ formic acid over $12 \mathrm{~min}$ at $40 \mu \mathrm{l} / \mathrm{min}$. Both the trapping and LC separation were performed at $1^{\circ} \mathrm{C}$ to minimize back exchange. Postprocessing was performed using Proteinlynx Global Server 3.0.3 and Dynamx 3.0 software to determine the average deuterium uptake for each peptide at each time point. For comparative analyses, the relative fractional uptake was determined by dividing the observed deuterium uptake by the number of available amide exchangers on each peptide.

\section{SUPPLEMENTARY MATERIALS}

Supplementary material for this article is available at https://science.org/doi/10.1126/ sciadv.abj4461

View/request a protocol for this paper from Bio-protocol.

\section{REFERENCES AND NOTES}

1. Y. Diekmann, J. B. Pereira-Leal, Evolution of intracellular compartmentalization. Biochem. J. 449, 319-331 (2013).

2. C. A. Kerfeld, S. Heinhorst, G. C. Cannon, Bacterial microcompartments. Annu. Rev. Microbiol. 64, 391-408 (2010).

3. W. Bonacci, P. K. Teng, B. Afonso, H. Niederholtmeyer, P. Grob, P. A. Silver, D. F. Savage, Modularity of a carbon-fixing protein organelle. Proc. Natl. Acad. Sci. U.S.A. 109, 478-483 (2012).

4. C. V. Iancu, D. M. Morris, Z. Dou, S. Heinhorst, G. C. Gordon, G. J. Jensen, Organization, structure, and assembly of $\alpha$-carboxysomes determined by electron cryotomography of intact cells. J. Mol. Biol. 396, 105-117 (2010).

5. T. O. Yeates, C. A. Kerfeld, S. Heinhorst, G. C. Cannon, J. M. Shively, Protein-based organelles in bacteria: Carboxysomes and related microcompartments. Nat. Rev. Microbiol. 6, 681-691 (2008).

6. S. C. Andrews, The Ferritin-like superfamily: Evolution of the biological iron storeman from a rubrerythrin-like ancestor. Biochim. Biophys. Acta 1800, 691-705 (2010).

7. E. Chiancone, P. Ceci, A. Ilari, F. Ribacchi, S. Stefanini, Iron and proteins for iron storage and detoxification. Biometals 17, 197-202 (2004).

8. M. Sutter, D. Boehringer, S. Gutmann, S. Günther, D. Prangishvili, M. J. Loessner, K. O. Stetter, E. Weber-Ban, N. Ban, Structural basis of enzyme encapsulation into a bacterial nanocompartment. Nat. Struct. Mol. Biol. 15, 939-947 (2008).

9. T. W. Giessen, P. A. Silver, Widespread distribution of encapsulin nanocompartments reveals functional diversity. Nat. Microbiol. 2, 17029 (2017).

10. D. He, S. Hughes, S. Vanden-Hehir, A. Georgiev, K. Altenbach, E. Tarrant, C. L. Mackay, K. J. Waldron, D. J. Clarke, J. Marles-Wright, Structural characterization of encapsulated ferritin provides insight into iron storage in bacterial nanocompartments. elife 5, e18972 (2016).

11. T. W. Giessen, B. J. Orlando, A. A. Verdegaal, M. G. Chambers, J. Gardener, D. C. Bell, G. Birrane, M. Liao, P. A. Silver, Large protein organelles form a new iron sequestration system with high storage capacity. eLife 8, e46070 (2019).

12. C. A. McHugh, J. Fontana, D. Nemecek, N. Cheng, A. A. Aksyuk, J. B. Heymann, D. C. Winkler, A. S. Lam, J. S. Wall, A. C. Steven, E. Hoiczyk, A virus capsid-like nanocompartment that stores iron and protects bacteria from oxidative stress. $E M B O \mathrm{~J}$. 33, 1896-1911 (2014)

13. F. Akita, K. T. Chong, H. Tanaka, E. Yamashita, N. Miyazaki, Y. Nakaishi, M. Suzuki, K. Namba, Y. Ono, T. Tsukihara, A. Nakagawa, The crystal structure of a virus-like particle from the hyperthermophilic archaeon Pyrococcus furiosus provides insight into the evolution of viruses. J. Mol. Biol. 368, 1469-1483 (2007).

14. A. Tamura, Y. Fukutani, T. Takami, M. Fujii, Y. Nakaguchi, Y. Murakami, K. Noguchi, M. Yohda, M. Odaka, Packaging guest proteins into the encapsulin nanocompartment from Rhodococcus erythropolis N771. Biotechnol. Bioeng. 112, 13-20 (2015).
15. D. He, C. Piergentili, J. Ross, E. Tarrant, L. R. Tuck, C. L. Mackay, Z. Mclver, K. J. Waldron, D. J. Clarke, J. Marles-Wright, Conservation of the structural and functional architecture of encapsulated ferritins in bacteria and archaea. Biochem. J. 476, 975-989 (2019).

16. R. Rahmanpour, T. D. H. Bugg, Assembly in vitro of Rhodococcus jostii RHA1 encapsulin and peroxidase DypB to form a nanocompartment. FEBS J. 280, 2097-2104 (2013).

17. J. Snijder, O. Kononova, I. M. Barbu, C. Uetrecht, W. F. Rurup, R. J. Burnley, M. S. T. Koay, J. J. L. M. Cornelissen, W. H. Roos, V. Barsegov, G. J. L. Wuite, A. J. R. Heck, Assembly and mechanical properties of the cargo-free and cargo-loaded bacterial nanocompartment encapsulin. Biomacromolecules 17, 2522-2529 (2016).

18. M. P. Andreas, T. W. Giessen, Large-scale computational discovery and analysis of virus-derived microbial nanocompartments. Nat. Commun. 12, 4748 (2021).

19. M. C. Jenkins, S. Lutz, Encapsulin nanocontainers as versatile scaffolds for the development of artificial metabolons. ACS Synth. Biol. 10, 857-869 (2021).

20. Y. H. Lau, T. W. Giessen, W. J. Altenburg, P. A. Silver, Prokaryotic nanocompartments form synthetic organelles in a eukaryote. Nat. Commun. 9, 1311 (2018).

21. F. Sigmund, S. Pettinger, M. Kube, F. Schneider, M. Schifferer, S. Schneider, M. V. Efremova, J. Pujol-Martí, M. Aichler, A. Walch, T. Misgeld, H. Dietz, G. G. Westmeyer, Iron-sequestering nanocompartments as multiplexed electron microscopy gene reporters. ACS Nano 13, 8114-8123 (2019).

22. S. Recalcati, E. Gammella, P. Buratti, G. Cairo, Molecular regulation of cellular iron balance. IUBMB Life 69, 389-398 (2017).

23. Y. Tang, A. Mu, Y. Zhang, S. Zhou, W. Wang, Y. Lai, X. Zhou, F. Liu, X. Yang, H. Gong, Q. Wang, Z. Rao, Cryo-EM structure of Mycobacterium smegmatis DyP-loaded encapsulin. Proc. Natl. Acad. Sci. U.S.A. 118, e2025658118 (2021).

24. X. Xiong, C. Sun, F. S. Vago, T. Klose, J. Zhu, W. Jiang, Cryo-EM structure of heterologous protein complex loaded Thermotoga Maritima encapsulin capsid. Biomolecules 10, 1342 (2020).

25. R. M. Putri, C. Allende-Ballestero, D. Luque, R. Klem, K.-A. Rousou, A. Liu, C. H.-H. Traulsen, W. F. Rurup, M. S. T. Koay, J. R. Castón, J. J. L. M. Cornelissen, Structural characterization of native and modified encapsulins as nanoplatforms for in vitro catalysis and cellular uptake. ACS Nano 11, 12796-12804 (2017).

26. J. Ross, T. Lambert, C. Piergentili, D. He, K. J. Waldron, C. L. Mackay, J. Marles-Wright, D. J. Clarke, Mass spectrometry reveals the assembly pathway of encapsulated ferritins and highlights a dynamic ferroxidase interface. Chem. Commun. 56, 3417-3420 (2020).

27. J. Zivanov, T. Nakane, B. O. Forsberg, D. Kimanius, W. J. H. Hagen, E. Lindahl, S. H. W. Scheres, New tools for automated high-resolution cryo-EM structure determination in RELION-3. elife 7, e42166 (2018).

28. R. J. Nichols, B. LaFrance, N. R. Phillips, D. R. Radford, L. M. Oltrogge, L. E. Valentin-Alvarado, A. J. Bischoff, E. Nogales, D. F. Savage, Discovery and characterization of a novel family of prokaryotic nanocompartments involved in sulfur metabolism. eLife 10, e59288 (2020).

29. B. LaFrance, C. Cassidy-Amstutz, R. J. Nichols, L. M. Oltrogge, E. Nogales, D. F. Savage, The encapsulin from Thermatoga maritima is a flavoprotein with a symmetry matched ferritin-like cargo protein. Sci. Rep. 11, 22810 (2021).

30. E. M. Williams, S. M. Jung, J. L. Coffman, S. Lutz, Pore engineering for enhanced mass transport in encapsulin nanocompartments. ACS Synth. Biol. 7, 2514-2517 (2018).

31. A. Punjani, D. J. Fleet, 3D variability analysis: Resolving continuous flexibility and discrete heterogeneity from single particle cryo-EM. J. Struct. Biol. 213, 107702 (2021).

32. L. Adamson, N. Tasneem, M. P. Andreas, W. Close, E. N. Jenner, T. N. Szyszka, R. Young L. C. Cheah, A. Norman, H. I. MacDermott-Opeskin, M. L. O'Mara, F. Sainsbury, T. W. Giessen, Y. H. Lau, Pore structure controls stability and molecular flux in engineered protein cages. bioRxiv 2021.01.27.428512 [Preprint]. 19 June 2021. https://doi.org/10.1101/2021.01.27.428512.

33. C. Piergentili, J. Ross, D. He, K. J. Gallagher, W. A. Stanley, L. Adam, C. L. Mackay, A. Baslé, K. J. Waldron, D. J. Clarke, J. Marles-Wright, Dissecting the structural and functional roles of a putative metal entry site in encapsulated ferritins. J. Biol. Chem. 295, 15511-15526 (2020).

34. J. Schindelin, I. Arganda-Carreras, E. Frise, V. Kaynig, M. Longair, T. Pietzsch, S. Preibisch C. Rueden, S. Saalfeld, B. Schmid, J.-Y. Tinevez, D. J. White, V. Hartenstein, K. Eliceiri, P. Tomancak, A. Cardona, Fiji: An open-source platform for biological-image analysis. Nat. Methods 9, 676-682 (2012).

35. S. V. Iverson, T. L. Haddock, J. Beal, D. M. Densmore, CIDAR MoClo: Improved MoClo assembly standard and new $E$. coli part library enables rapid combinatorial design for synthetic and traditional biology. ACS Synth. Biol. 5, 99-103 (2015).

36. F. Studier, Protein production by auto-induction in high-density shaking cultures. Protein Expr. Purif. 41, 207-234 (2005)

37. S. Q. Zheng, E. Palovcak, J.-P. Armache, K. A. Verba, Y. Cheng, D. A. Agard, MotionCor2: Anisotropic correction of beam-induced motion for improved cryo-electron microscopy. Nat. Methods 14, 331-332 (2017).

38. A. Rohou, N. Grigorieff, CTFFIND4: Fast and accurate defocus estimation from electron micrographs. J. Struct. Biol. 192, 216-221 (2015). 
39. A. Punjani, J. L. Rubinstein, D. J. Fleet, M. A. Brubaker, cryoSPARC: Algorithms for rapid unsupervised cryo-EM structure determination. Nat. Methods 14, 290-296 (2017).

40. T. D. Goddard, C. C. Huang, E. C. Meng, E. F. Pettersen, G. S. Couch, J. H. Morris, T. E. Ferrin, UCSF ChimeraX: Meeting modern challenges in visualization and analysis. Protein Sci. 27, 14-25 (2018).

41. L. A. Kelly, S. Mezulis, C. Yates, M. Wass, M. Sternberg, The Phyre2 web portal for protein modeling, prediction and analysis. Nat. Protoc. 10, 845-858 (2015).

42. E. F. Pettersen, T. D. Goddard, C. C. Huang, E. C. Meng, G. S. Couch, T. I. Croll, J. H. Morris, T. E. Ferrin, UCSF ChimeraX: Structure visualization for researchers, educators, and developers. Protein Sci. 30, 70-82 (2021).

43. P. V. Afonine, B. K. Poon, R. J. Read, O. V. Sobolev, T. C. Terwilliger, A. Urzhumtsev P. D. Adams, Real-space refinement in PHENIX for cryo-EM and crystallography. Acta Crystallogr. D Struct. Biol. 74, 531-544 (2018).

44. P. Emsley, B. Lohkamp, W. G. Scott, K. Cowtan, Features and development of Coot. Acta Crystallogr. D Biol. Crystallogr. 66, 486-501 (2010).

45. V. B. Chen, W. B. Arendall, J. J. Headd, D. A. Keedy, R. M. Immormino, G. J. Kapral, L. W. Murray, J. S. Richardson, D. C. Richardson, MolProbity: All-atom structure validation for macromolecular crystallography. Acta Crystallogr. D Biol. Crystallogr. 66, 12-21 (2010).

46. P. V. Afonine, B. P. Klaholz, N. W. Moriarty, B. K. Poon, O. V. Sobolev, T. C. Terwilliger, P. D. Adams, A. Urzhumtsev, New tools for the analysis and validation of cryo-EM maps and atomic models. Acta Crystallogr. D Struct. Biol. 74, 814-840 (2018).

47. B. A. Barad, N. Echols, R. Y.-R. Wang, Y. Cheng, F. DiMaio, P. D. Adams, J. S. Fraser, EMRinger: Side chain-directed model and map validation for 3D cryo-electron microscopy. Nat. Methods 12, 943-946 (2015).

48. F. Sievers, D. G. Higgins, Clustal Omega, accurate alignment of very large numbers of sequences. Methods Mol. Biol. 1079, 105-116 (2014).

49. P. Gouet, X. Robert, E. Courcelle, ESPript/ENDscript: Extracting and rendering sequence and 3D information from atomic structures of proteins. Nucleic Acids Res. 31, 3320-3323 (2003)

50. G. R. Masson, J. E. Burke, N. G. Ahn, G. S. Anand, C. Borchers, S. Brier, G. M. Bou-Assaf, J. R. Engen, S. W. Englander, J. Faber, R. Garlish, P. R. Griffin, M. L. Gross, M. Guttman, Y. Hamuro, A. J. R. Heck, D. Houde, R. E. lacob, T. J. D. Jørgensen, I. A. Kaltashov, J. P. Klinman, L. Konermann, P. Man, L. Mayne, B. D. Pascal, D. Reichmann, M. Skehel, J. Snijder, T. S. Strutzenberg, E. S. Underbakke, C. Wagner, T. E. Wales, B. T. Walters, D. D. Weis, D. J. Wilson, P. L. Wintrode, Z. Zhang, J. Zheng, D. C. Schriemer, K. D. Rand, Recommendations for performing, interpreting and reporting hydrogen deuterium exchange mass spectrometry (HDX-MS) experiments. Nat. Methods 16, 595-602 (2019).

51. M. Laguerre, M. Saux, J. P. Dubost, A. Carpy, in Pharmaceutical Sciences (John Wiley \& Sons Ltd., 1997), vol. 3, pp. 217-222.

Acknowledgments: We acknowledge Diamond Light Source for access and support of the cryo-EM facilities at the UK's National Electron Bio-imaging Centre (eBIC) (under proposal EM16637), funded by the Wellcome Trust, MRC, and BBRSC. We thank Y. Song for their assistance at $\mathrm{eBIC}$ with sample loading and data collection setup. This research made use of the Rocket High Performance Computing service at Newcastle University; we thank K. Bower for assistance with the use of the Rocket service. Negative-stain TEM was performed in Edinburgh Electron Microscopy Laboratory and the Newcastle University Electron Microscopy Research Services; we thank S. Mitchell, T. Davey, and R. Laws for their technical support. Cryo-EM grid screening was performed in the Edinburgh cryo-EM facility in School of Biological Sciences at the University of Edinburgh. The cryo-EM facility was set up with funding from the Wellcome Trust (087658/Z/08/Z) and SULSA and is supported by the Wellcome Centre for Cell Biology. We thank M. Noble for donating GPU time to our CryoSPARC analyses. Funding: This work was supported by a Royal Society Research Grant awarded to J.M.-W. (RG130585) and a BBSRC New Investigator Grant to J.M.-W. and D.J.C. (BB/N005570/1). C.P. was funded by the BBSRC New Investigator Grant (BB/N005570/1). J.M.-W. is funded by Newcastle University. D.J.C., T.L., and J.R. are funded by the University of Edinburgh. J.R. was funded by a BBSRC EastBio DTP studentship (BB/M010996/1). Z.M. is funded by a BBSRC NLD DTP studentship (BB/M011186/1). J.E.B. was funded by DSTL via Imperial College. E.Z.-A. was funded by an IBiolC PhD studentship with Fujifilm Diosynth Biotechnologies. K.J.W. was funded by the Biotechnology and Biological Sciences Research Council (BB/S006818/1). Equipment for transmission electron microscopy at Newcastle University was funded through the BBSRC 17ALERT call (BB/R013942/1). Equipment for mass spectrometry at the University of Edinburgh was funded through the BBSRC 17ALERT call (BB/R013993/1). The work of M.D.W. is supported by the Wellcome Trust and Royal Society (210493), Medical Research Council (T029471/1), and the University of Edinburgh. The Wellcome Centre for Cell Biology is supported by core funding from the Wellcome Trust (203149). Author contributions: Conceptualization: J.R., J.M.-W., and D.J.C. Methodology: J.R., Z.M., T.L., C.P., A.B., J.M.-W., and D.J.C. Validation: J.R., Z.M., T.L., J.M.-W., and D.J.C.. Formal analysis: J.R., Z.M., T.L., A.B., P.J., J.M.-W., and D.J.C. Investigation: J.R., Z.M., K.J.G., J.E.B., C.P., and E.Z.-A. Resources: K.J.G., F.L.C. C.L.M., J.E.B., A.B., E.Z.-A., and M.D.W. Data curation: J.R., T.L., D.J.C., A.B., and J.M.-W. Writingoriginal draft preparation: J.R., D.J.C., and J.M.-W. Writing-review and editing: J.R., Z.M., C.P., A.B., M.D.W., K.J.W., J.M.-W., and D.J.C. Visualization: J.R., Z.M., T.L., D.J.C., and J.M.-W. Supervision: J.M.-W. and D.J.C. Funding acquisition: M.D.W., L.E.H., K.J.W., D.J.C., and J.M.-W. Competing interests: The authors declare that they have no competing interests. Data and materials availability: All data needed to evaluate the conclusions in the paper are present in the paper and/or the Supplementary Materials, or have been deposited in appropriate publicly available data repositories. Cryo-electron microscopy movie stacks are available at the EMPIAR database (EMPIAR-1218). Single-particle reconstructions have been deposited at the EMDB (entries: EMD-12853, EMD-12873, EMD-12859, and EMD-12864); derived atomic models are deposited at the PDB (entries: 7OE2 and 7OEU). Enzymatic assay data and negative-stain electron micrographs are deposited at Figshare.

Submitted 14 May 2021

Accepted 2 December 2021

Published 26 January 2022

10.1126/sciadv.abj4461 


\section{ScienceAdvances}

\section{Pore dynamics and asymmetric cargo loading in an encapsulin nanocompartment}

Jennifer RossZak MclverThomas LambertCecilia PiergentiliJasmine Emma BirdKelly J. GallagherFaye L.

CruickshankPatrick JamesEfrain Zarazúa-ArvizuLouise E. HorsfallKevin J. WaldronMarcus D. WilsonC. Logan

MackayArnaud BasléDavid J. ClarkeJon Marles-Wright

Sci. Adv., 8 (4), eabj4461. • DOI: 10.1126/sciadv.abj4461

View the article online

https://www.science.org/doi/10.1126/sciadv.abj4461

Permissions

https://www.science.org/help/reprints-and-permissions 